\title{
Coumarin antifungal lead compounds, predicted mechanism of action
}

Robert Kingsford-Adaboh ${ }^{1}$, Daniel Moscoh ${ }^{2}$, Johannes Reynisson ${ }^{2}$

${ }^{1}$ Department Of Chemistry, University Of Ghana, Accra, Ghana, ${ }^{2}$ School of Chemical Sciences, University of Auckland, Auckland, New Zealand

E-mail: kadabohs@yahoo.com

Introduction: Candida albicans is one of the most common causative fungi infection despite major efforts to control it. C. albicans has emerged as one of the main causes of morbidity and mortality in immunocompromised patients suffering from, e.g., cancer or AIDS. Drug -resistance on existing drugs calls for need for new antifungal drugs. Hence it is necessary to develop new antifungal drugs to address these emerging challenges. Fungal pathogens continue to pose challenges to humans and plants despite efforts to control them. Two coumarins, robustic acid and thonningine- $\mathrm{C}$ isolated from Millettia thonningii, show promising activity against the fungus Candida albicans with minimum fungicidal concentration of 1.0 and 0.5 $\mathrm{mg} / \mathrm{mL}$, respectively. Molecular modelling against the putative bio-molecular target, lanosterol 14_-demethylase (CYP51), revealed a plausible binding mode for the active compounds, in which the hydroxyl group binds with a methionine backbone carboxylic group blocking access to the iron catalytic site. This binding disrupts the synthesis of several important sterols for the survival of fungi.

Method and Results: Compounds from seedsof M.thonningii were isolated and activity investigated C. albicans. Isolation yeilded robustic acid (1), thonningine-C (2), alpinumisoflavone, O'O-dimethylalpinumisoflavone.

The compounds were tested against the wild C. albicans strain and a reference strain ATCC18804. The agar well diffusion method was used to evaluate zones of inhibition (ZOI). Thermochemical calculations using Gausian 09 software suite. The predicted mechanism and bioassay results show that robustic acid (1), thonningine- $C$ (2) were active against the fungal strains tested.

2) Daniel M. Ayine-Tora, Robert Kingsford-Adaboh, William A. Asomaning, Jerry J.E.K. Harrison, Felix C. Mills-Robertson, Yahaya Bukari, Patrick O. Sakyi, Sylvester Kaminta and Jóhannes Reynisson (2016): Coumarin Antifungal Lead Compounds from Millettia thonningii and Their Predicted Mechanism of Action: Molecules, 21, 1369; doi:10.3390/molecules21101369

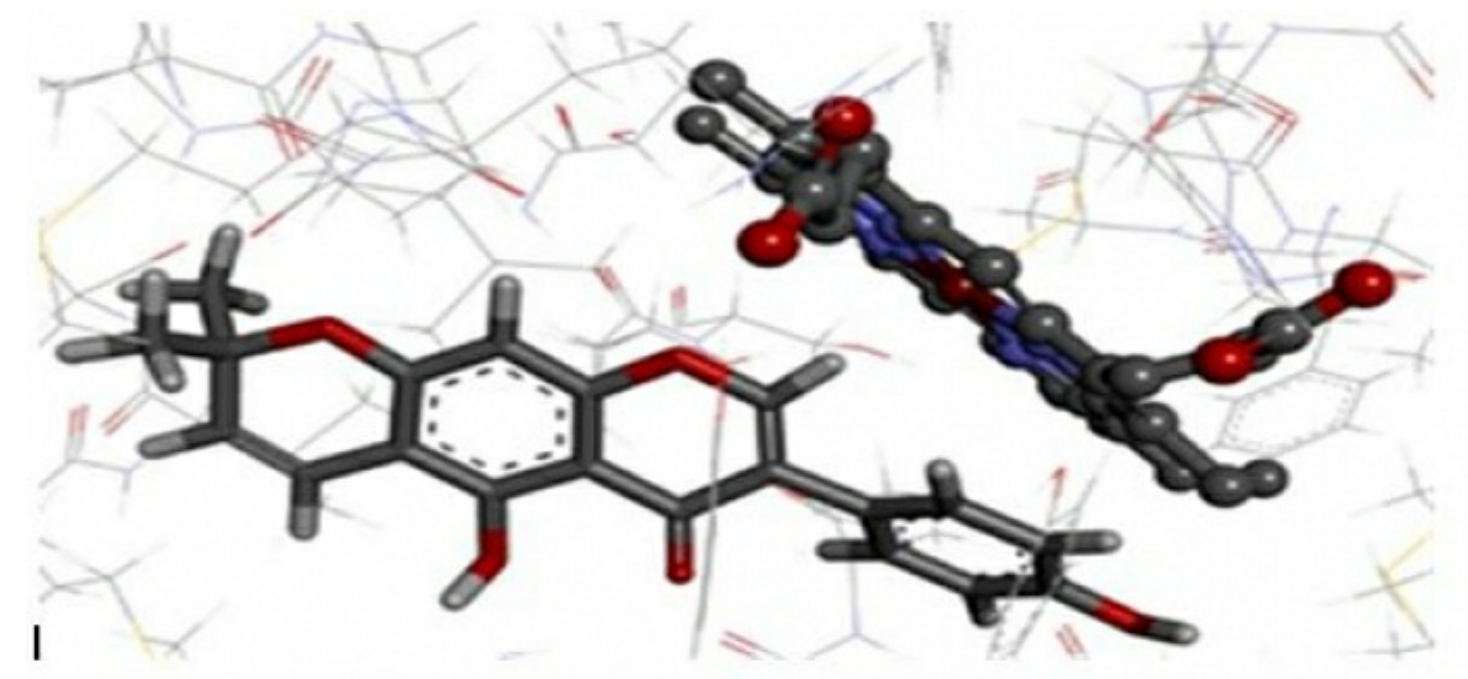

Keywords: Candida albicans; molecular modelling, isoflavones 